\title{
Optical/UV and X-Ray Microwave Kinetic Inductance Strip Detectors
}

\author{
B.A. Mazin • M.E. Eckart • B. Bumble • \\ S. Golwala $\cdot$ P.K. Day $\cdot$ J. Gao $\cdot$ J. Zmuidzinas
}

Received: 23 July 2007 / Accepted: 15 September 2007 / Published online: 9 January 2008 (C) U.S. Government 2008

\begin{abstract}
Microwave Kinetic Inductance Detectors (MKIDs) are superconducting detectors that sense the change in the surface impedance of a thin superconducting film when Cooper Pairs are broken by using a high quality factor resonant circuit. We are developing strip detectors that have aluminum MKID sensors on both ends of a rectangular tantalum strip. These devices can provide one dimensional spatial imaging with high quantum efficiency, energy resolution, and microsecond time resolution for single photons from the IR to the X-ray. We have demonstrated X-ray strip detectors with an energy resolution of $62 \mathrm{eV}$ at $6 \mathrm{keV}$, and hope to improve this substantially. We will also report on our progress towards optical arrays for a planned camera for the Palomar 200" telescope.
\end{abstract}

Keywords Microwave $\cdot$ Kinetic $\cdot$ Inductance $\cdot$ Detector $\cdot$ MKID $\cdot$ LTD

\section{Introduction}

A Microwave Kinetic Inductance Detector (MKID) [1, 5] is essentially a high-Q resonant circuit made out of either superconducting microwave transmission lines or a lumped element LC resonator. Photons or particles hitting an MKID break Cooper Pairs (creating free charge carriers called quasiparticles) which changes the surface impedance of the transmission line or inductive element. This causes the resonant frequency and quality factor to shift an amount proportional to the energy deposited by the photon or particle.

B.A. Mazin $(\varangle) \cdot$ M.E. Eckart · B. Bumble · S. Golwala · P.K. Day · J. Gao · J. Zmuidzinas Jet Propulsion Lab, California Institute of Technology, Pasadena, CA 91109, USA

e-mail: ben.mazin@jpl.nasa.gov 


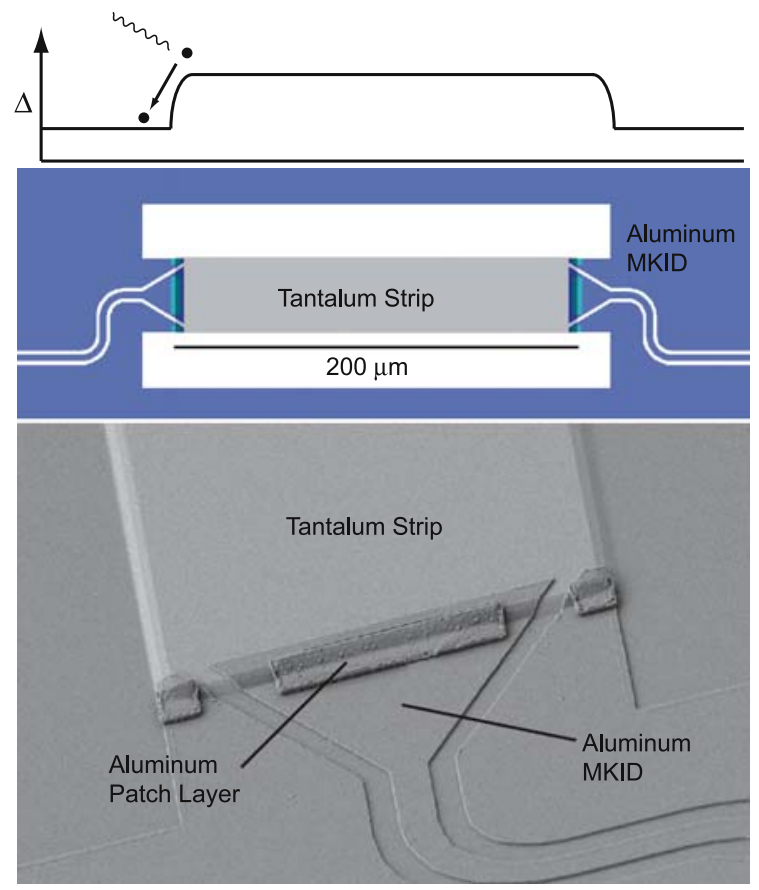

Fig. 1 (Color online) The middle panel, Fig. 1b contains a drawing of the central region of a MKID strip detector. A $200 \times 35 \mu \mathrm{m}, 600 \mathrm{~nm}$ thick tantalum strip $(\mathrm{RRR}=22.6)$ is fabricated on R-plane sapphire and has MKIDs attached to both ends. The $3 \mu \mathrm{m}$ center strip of the $200 \mathrm{~nm}$ thick aluminum $(\mathrm{RRR}=9.5) \mathrm{CPW}$ resonator that comprises the MKID is flared out where it contacts the tantalum strip to allow lateral trapping of quasiparticles. The top panel, Fig. 1a shows the superconducting gap $\Delta$ of the structure, including a quasiparticle diffusing into the aluminum MKID and being trapped by phonon emission. The bottom panel, Fig. 1c shows a SEM of the Al-Ta interface from the wafer tested in this paper. A patch of aluminum patterned with a liftoff process is used to bridge the Al-Ta interface to avert a step coverage problem. In this device the tantalum is nicely sloped and the aluminum resonator climbs smoothly over the step

We have demonstrated the detection of submillimeter, optical/UV, and X-ray photons with MKIDs. In this paper we will detail our efforts to build large arrays of MKIDs for X-ray and Optical/UV astronomy. The basis of our work is the tantalum/aluminum strip detector [2, 7]. In this structure, shown in Fig. 1, photons are absorbed in a tantalum absorbing strip. The quasiparticles created by the photon diffuse from the absorption site until they reach the ends of the strip. At the ends they enter a lower gap aluminum trapping bar that is also the center strip of a $1 / 2$-wave coplanar waveguide (CPW) MKID. In this bar they quickly emit a phonon and relax to the gap energy of aluminum, preventing their return to the tantalum absorber. The change in the surface impedance caused by the excess quasiparticles modifies probe signals sent down the feedline. The modified signal is measured for both MKIDs. The signals are summed to provide the total photon energy, and the position is available by taking the ratio of the signals. This can be represented as a scatter plot (Fig. 3). Following Kraus [4] we can write an expression for the fraction of the total created quasiparticles $J_{0}$ that reach the end of the strip $J\left(x_{0}\right)=J_{0} \frac{\sinh \left(\beta x_{0} / L\right)}{\sinh (\beta)}$ where $x_{0}$ is the distance away from the end of the strip that the photon hit, and $L$ is the length of the 
Fig. 2 (Color online) The effect of loss in a strip detector. The $x$-axis and $y$-axis show the normalized amount of quasiparticles that reach the ends of the strip for a given photon absorbtion position for various loss factors $\beta$. For example, an X-ray event in the center of a strip with $\beta=3$ will deliver $20 \%$ of the total quasiparticles to each end of the strip. The remaining $60 \%$ will be lost to recombination

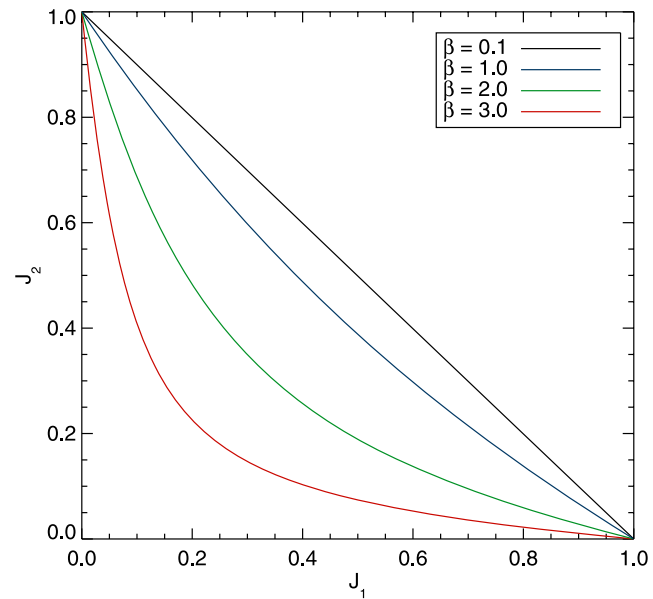

strip. The parameter $\beta$ is related to the diffusion constant $D$, quasiparticle recombination time $\tau_{q p}$, and the length of the strip $L$ by $\beta=L / \sqrt{D \tau_{q p}}$.

\section{X-ray Detectors}

We have fabricated X-ray strip detectors with a $600 \mathrm{~nm}$ thick tantalum absorber and $200 \mathrm{~nm}$ thick aluminum MKIDs, as shown in Fig. 1c. A scatter plot of events generated by illuminating the device in Fig. $1 \mathrm{~b}$ with an ${ }^{55} \mathrm{Fe} \mathrm{X}$-ray source yields Fig. 3. The Mn $K_{\alpha}$ and $K_{\beta}$ lines from the ${ }^{55} \mathrm{Fe}$ source are clearly visible. Analysis of this data yields an energy resolution of $66 \mathrm{eV}$ at $6 \mathrm{keV}$, which is approximately the same as the value we expect from noise measurements. By modeling photon events from various locations along the strip we measure a tantalum diffusion constant of $13.5 \pm 1.8 \mathrm{~cm}^{2} / \mathrm{sec}$ and a tantalum quasiparticle lifetime of $34.5 \pm 5.7 \mu \mathrm{s}$. These parameters allow us to calculate the tantalum diffusion length $l_{\mathrm{Ta}}=\sqrt{D_{\mathrm{Ta}} \tau_{\mathrm{Ta}}}=216 \pm 30 \mu \mathrm{m}$. For more details see [2, 7].

The X-ray detectors shown here are limited by the poor quality of the sapphire used as a substrate. We believe we are getting excess phase noise from two-level systems (TLS) in the dielectric [3]. We have measured similar devices on higher quality sapphire that should improve our energy resolution to $12 \mathrm{eV}$ with the same geometry detector. Higher quality substrates, optimizing the resonator geometry, using an amplitude readout, and using different superconductors may improve this further.

\section{Optical/UV Detectors}

While the extremely interesting X-ray astronomy [2] delivered with X-ray MKIDs is only available with a space-based telescope, optical/UV detectors provide exciting science opportunities from the ground and from space $[6,9,10]$.

Adapting our strip detectors for the optical requires boosting their responsivity by a $1000 \times$, although the final signal to noise requirements are significantly lower. 


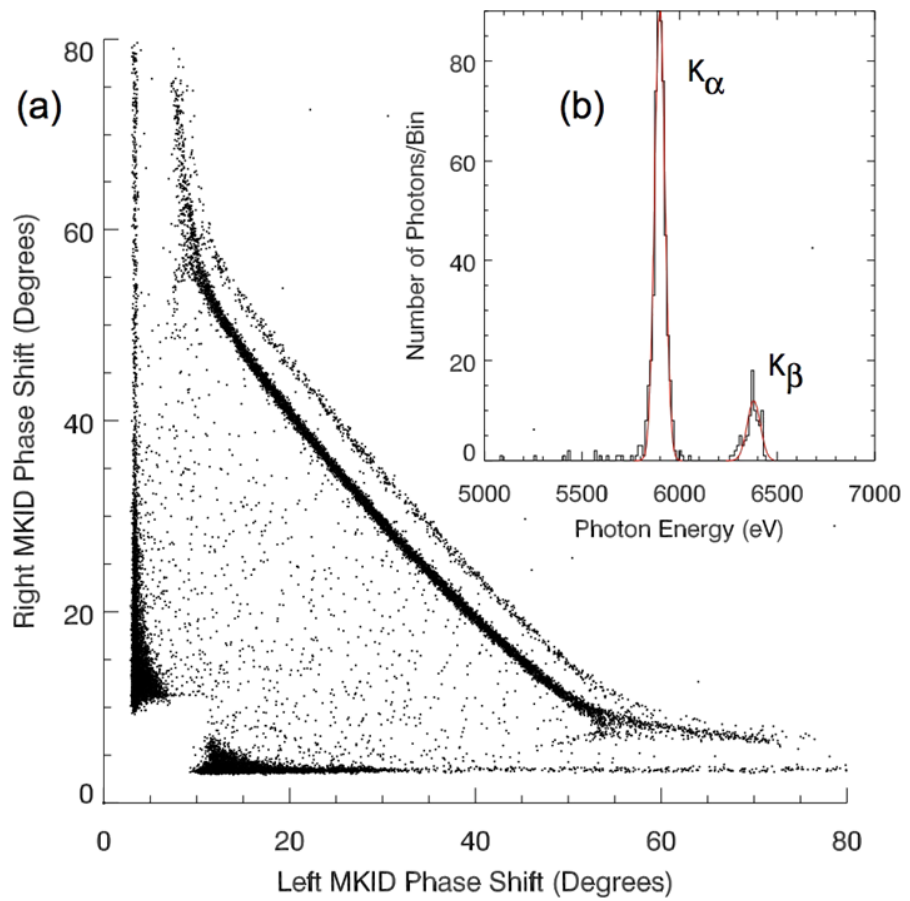

Fig. 3 (Color online) The optimally filtered maximum phase pulse height in degrees observed in an aluminum MKIDs attached to a $200 \mu \mathrm{m}$ tantalum strip is shown in Fig. 3a. The pulse height in the left MKID is shown on the $x$-axis, while the right MKID is shown on the $y$-axis. The Mn $K_{\alpha}$ and $K_{\beta}$ lines from the ${ }^{55} \mathrm{Fe}$ source are clearly visible. This data is fit to determine the diffusion length, and this is used to compute the energy spectrum shown in the inset, Fig. 3b. We calculate a FWHM energy width $\delta E=62 \mathrm{eV}$ at $5.899 \mathrm{keV}$ when we restrict our data to all pulses that show greater than 22 degrees of phase shift in both MKIDs

This can be achieved by making the aluminum MKIDs thinner ( $20 \mathrm{~nm}$ vs. $200 \mathrm{~nm}$ ), smaller ( $1 \mu \mathrm{m}$ wide vs. $3 \mu \mathrm{m}$ wide), and increasing the quality factor $Q$ by a factor of 3 or more.

In order to stack the strips detectors closer together and ensure a electrically contiguous ground plane, we have developed a new coupling system that uses a microstrip feedline and coupler. We use standard CPW bond pads, but use a CPW to microstrip transition structure to move to a $2 \mu \mathrm{m}$ wide microstrip feedline as shown in Fig. 4a. The coupling structure uses parallel lines in the center strip of a CPW resonator as shown in Fig. 4b. This feedline and coupler structure has performed well in actual devices.

Our optical/UV test devices contain 50 strips, each with a width of $20 \mu \mathrm{m}$ and lengths varying from 100-800 $\mu \mathrm{m}$. The strips have $2 \mu \mathrm{m}$ gaps between them, allowing a filling factor of $90 \%$. They are fabricated in a seven layer process. First the tantalum absorber is deposited and patterned with sloped edges as described in [7]. $\mathrm{A} \mathrm{SiO}_{2}$ layer is deposited and pattered as a protection layer for the tantalum absorber. Aluminum is then deposited over the wafer and patterned into the last $\sim 100 \mu \mathrm{m}$ of the resonator's center strip. The niobium ground plane is deposited and etched in 

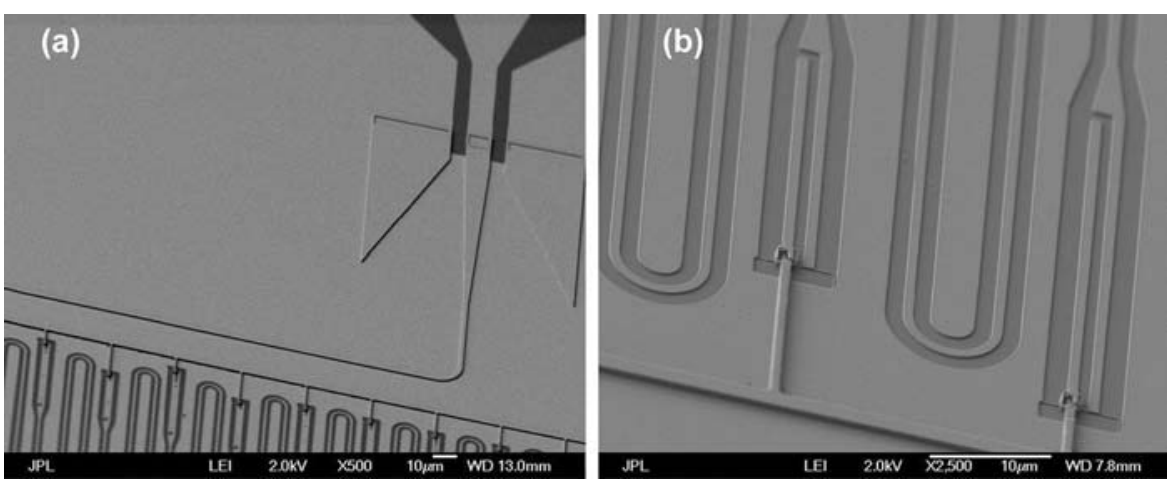

Fig. 4 Panel (a) shows the CPW to microstrip transition structure in the top right corner. A $2 \mu \mathrm{m}$ wide microstrip feedline exits the structure and is connected to the coupling structure shown in Panel (b) if each resonator

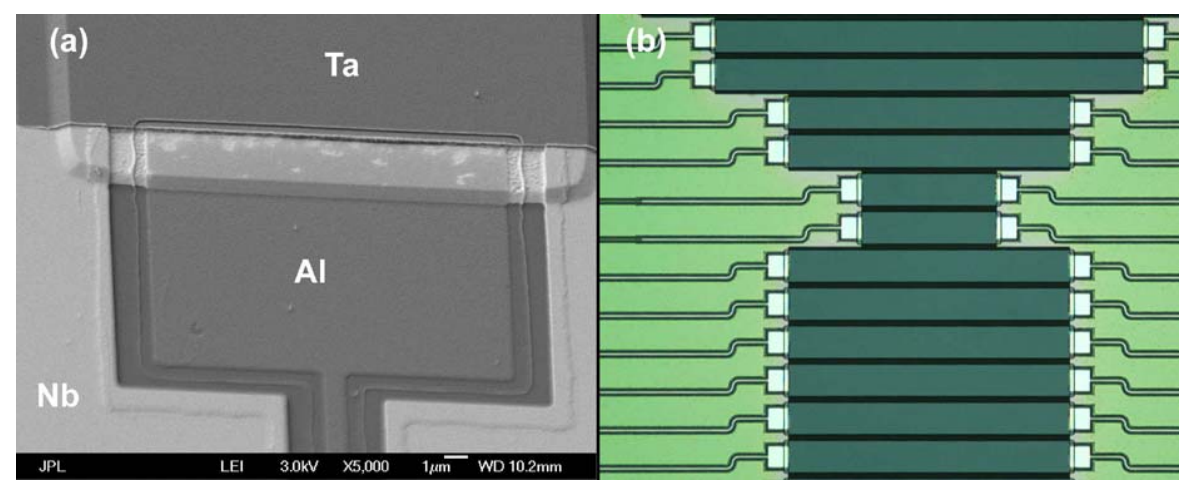

Fig. 5 (Color online) Panel (a) shows the trapping bar that traps quasiparticles at the end of the strips. Care must be taken in this area, and in this device some pitting and corrosion of the $20 \mathrm{~nm}$ aluminum film is evident. The various materials used in the construction are labeled in this figure. Panel (b) shows an optical micrograph of part of the array of 50 strips that comprise our test device

several key places. Next, a $\mathrm{SiO}_{2}$ microstrip dielectric layer is deposited and etched to make vias. This is followed by the niobium microstrip wiring layer. After the wiring layer is deposited the niobium ground plane layer is etched again to define the slots of the CPW resonators. It is important that this step is done near the end as we have found that reactive ion etches done after the slots are etched tends to degrade the quality of the resonators. As a final step a plasma etch removes any exposed $\mathrm{SiO}_{2}$ in order to eliminate the possibility it may contribute dielectric noise.

This device is very complex, and several of the steps can interact with each other in unexpected ways. We believe we have most of the problems solved and are currently testing a very promising strip detector.

Despite fabrication difficulties we have illuminated the individual resonators with $254 \mathrm{~nm}$ UV photons and seen excellent results. Figure 6 shows the pulse recorded when a photon hits the center aluminum strip of one of our resonators. The signal to noise of this event is as high as expected for thin devices on a high quality sapphire 


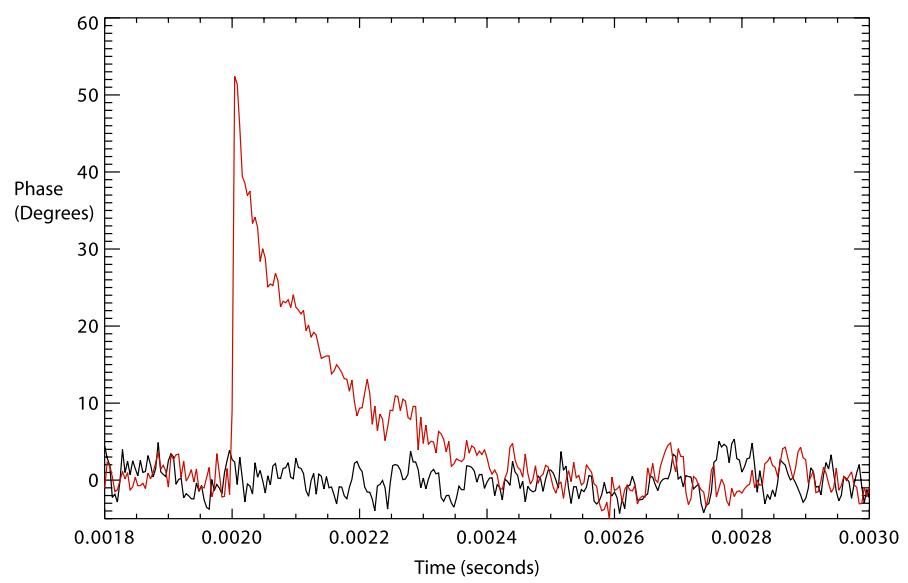

Fig. 6 (Color online) The response of a optical/UV MKID to $254 \mathrm{~nm}$ photon illumination is show in red. The black line is the response of a nearby resonator. This UV photon was most likely absorbed directly in the aluminum center strip, not in the tantalum absorber

substrate, and shows that we have the required sensitivity to make a scientifically interesting detector with no additional changes to the methods or materials.

\section{Conclusions}

Optical/UV/X-ray MKIDs are excellent candidates for large arrays for the future ground and space based missions. The designs presented here can provide very high fill factors in one dimension-for example, an optical/UV array with $1024 \times 20$ pixels would be very straightforward to fabricate with this style device. The size of the MKIDs compared with the pixels means that full 2-d arrays will have poor fill factors without the use of complex optics to slice up the focal plane. In the longer term, MKID arrays using microstrip MKIDs with thin-film dielectrics should allow us build near $100 \%$ fill factor megapixel scale imagers in the optical/UV. These arrays, coupled with the rapid development of digital readout techniques [8], allow us to contemplate extremely large arrays for the next generation of astronomical imaging spectrophotometers.

Acknowledgements We are grateful for the support of the Jet Propulsion Lab's Research and Technology Development (R\&TD) fund as well as the generous contributions of the Gordon and Betty Moore Foundation.

\section{References}

1. P. Day, H. Leduc, B. Mazin, A. Vayonakis, J. Zmuidzinas, A superconducting detector suitable for use in large arrays. Nature 425, 817-821 (2003)

2. M. Eckart, Measurements of X-ray selected agn and novel superconducting X-ray detectors. Ph.D. thesis, California Institute of Technology (2007) 
3. J. Gao, J. Zmuidzinas, B. Mazin, H. LeDuc, P. Day, Noise properties of superconducting coplanar waveguide microwave resonators. Appl. Phys. Lett. 90, 102507 (2007)

4. H. Kraus, F. Vonfeilitzsch, J. Jochum, R. Mossbauer, T. Peterreins, F. Robst, Quasiparticle trapping in a superconductive detector system exhibiting high-energy and position resolution. Phys. Lett. B 231, 195-202 (1989)

5. B. Mazin, Microwave kinetic inductance detectors. Ph.D. thesis, California Institute of Technology (2004)

6. B. Mazin, R. Brunner, Simulated extragalactic observations with a cryogenic imaging spectrophotometer. Astron. J. 120(5), 2721-2729 (2000)

7. B. Mazin, B. Bumble, P. Day, M. Eckart, S. Golwala, J. Zmuidzinas, F. Harrison, Position sensitive X-ray spectrophotometer using microwave kinetic inductance detectors. Appl. Phys. Lett. 89, 222507 (2006)

8. B. Mazin, P. Day, K. Irwin, C. Reintsema, J. Zmuidzinas, Digital readouts for large microwave lowtemperature detector arrays, in Proceeding of LTD-11. AIP Conference Proceedings, vol. 559 (2006), pp. 799-801

9. A. Reynolds, G. Ramsay, J. de Bruijne, M. Perryman, M. Cropper, C. Bridge, A. Peacock, High-speed, energy-resolved stj observations of the am her system v2301 oph. Astron. Astrophys. 435, 225-230 (2005)

10. R. Romani, A. Miller, B. Cabrera, S. Nam, J. Martinis, Phase-resolved crab studies with a cryogenic transition-edge sensor spectrophotometer. Astrophys. J. 563, 221-228 (2001) 